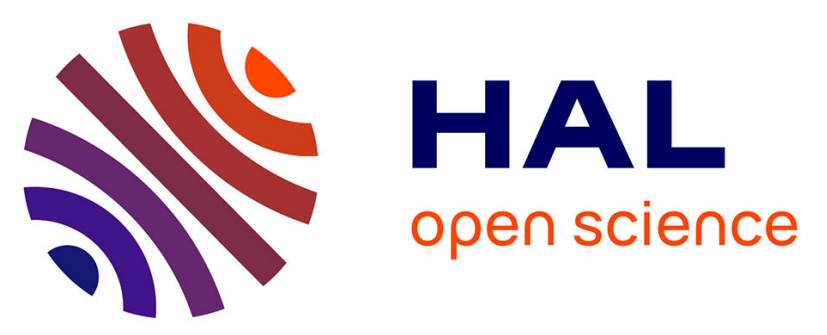

\title{
XANES STUDY OF SULFUR AND ZINC K-EDGES IN ZINCBLENDE: EXPERIMENTS AND MULTIPLE-SCATTERING CALCULATIONS
}

P. Sainctavit, J. Petiau, G. Calas, M. Benfatto, C. Natoli

\section{- To cite this version:}

P. Sainctavit, J. Petiau, G. Calas, M. Benfatto, C. Natoli. XANES STUDY OF SULFUR AND ZINC K-EDGES IN ZINCBLENDE: EXPERIMENTS AND MULTIPLE-SCATTERING CALCULATIONS. Journal de Physique Colloques, 1987, 48 (C9), pp.C9-1109-C9-1112. 10.1051/jphyscol:19879201 . jpa-00227320

\section{HAL Id: jpa-00227320 https://hal.science/jpa-00227320}

Submitted on 1 Jan 1987

HAL is a multi-disciplinary open access archive for the deposit and dissemination of scientific research documents, whether they are published or not. The documents may come from teaching and research institutions in France or abroad, or from public or private research centers.
L'archive ouverte pluridisciplinaire HAL, est destinée au dépôt et à la diffusion de documents scientifiques de niveau recherche, publiés ou non, émanant des établissements d'enseignement et de recherche français ou étrangers, des laboratoires publics ou privés. 


\title{
XANES STUDY OF SULFUR AND ZINC K-EDGES IN ZINCBLENDE : EXPERIMENTS AND MULTIPLE-SCATTERING CALCULATIONS
}

\author{
P. SAINCTAVIT****, J. PETIAU*, G. CALAS*, M. BENFATto** and \\ C.R. NATOLI*** \\ * Laboratoire de Minéralogie - Cristallographie. Universités \\ Paris VI et VII, 4, PIace Jussieu, F-75252 Paris Cedex 05 , \\ France \\ ** Saint-Gobain Recherche, 39, Quai Lucien Lefranc, \\ F-93304 Auberviliters Cedex, France \\ ** INFN Laboratori Nazionali di Frascati, I-00044 Frascati, \\ Italy
}

Résumé. Des calculs de diffusion mutliple ont été effectués aux seuils $\mathbf{K}$ du soufre et du zinc dans la blende et les résultats ont été comparés aux spectres expérimentaux. L'influence de la taille de l'amas a été spécialement étudiée. Les effets de structure de bande sont bien reproduits pour les amas de 99 atomes.

\begin{abstract}
Multiple scattering calculations were carried out at the sulphur and zinc K-edges of zincblende and the results compared to experimental spectra. A special attention was paid to the influence of the cluster size. With 99 atom clusters, band-structure effects are well reproduced.
\end{abstract}

\section{Introduction}

The XANES (X-ray Absorption Near-Edge Structure) of deep level spectra potentially contains structural and electronic information. In this energy range the single-scattering formulation (EXAFS) breaks down because the cross-section for electron scattering might be large. Two types of interpretation have been developed relying either on the use of multiplescattering (MS) theory(1-4) or on band structure calculations extended towards large kinetic energies of the photoelectron(5-6). Both methods have been used in few model compounds and have produced results consistent with the experimental spectra(1-9).

The band structure approach is not manageable but in perfect crystals with a few atoms in the Wigner cell. For molecular systems, for impurities in solid compounds or for surface atoms, the MS formulation is the only one tractable. Up to now all the calculations have been performed in the framework of the muffin-tin approximation. This approximation is well suited for ionic compounds and metals where the bondings are not directional. In covalent systems spherical potentials around each atom are expected to be a poor representation of the actual potential since they completely neglect the directionality of the chemical bonds.

In this paper we report MS calculations at the sulfur and zinc K-edges of zincblende and we compare the results with experimental spectra. This study enters our general interest for II-VI semiconductors containing cationic impurities. At the first step we have considered pure $\mathrm{ZnS}$.

\section{Experimental}

The spectra have been recorded in the transmission mode using the synchrotron radiation of LURE-DCI (ORSAY). A double-crystal monochromator was used with $\mathrm{Si}(111)$ reflections at the sulfur $\mathrm{K}$-edge $(2472 \mathrm{eV})$ and $\mathrm{Si}(400)$ reflections at the zinc K-edge $(9650 \mathrm{eV})$. At sulfur $\mathrm{K}$-edge the higher order contamination was suppressed by a specular reflection equipment(10).

\section{Theoretical}

Theoretical calculations were performed in the multiple-scattered-wave framework, developed by Natoli and coworkers, using the "extended continuum method" $(1-2)$. In this method the local potential is described in the muffin-tin approximation: inside every muffin-tin sphere the potential is spherically averaged and the interstitial region is not bounded by an outer-sphere. We did not use any Watson charge to neutralize the cluster charge or any Madelung potential to simulate the influence of the crystal around the cluster(1). For all energies greater than the interstitial muffin-tin potential the normalized final-state wave function is calculated by solving the multiple scattering equations. This "extended continum" method leads to results similar to SCF calculations for the bound states and requires much less time consuming calculations. The expansion of the final state wave function is made on a combination of real spherical harmonics centered on every atom. The combinations which belong to the $t_{2}$ representation were the only ones retained because they are the only dipole-allowed final states starting from an is initial state. The expansion was truncated at $l=3$ in the first $40 \mathrm{eV}$ and at $1=5$ in the next $30 \mathrm{eV}$.

For the construction of the Coulomb part of the muffin-tin potentials we chose atomic potentials calculated by Clementi and Roetti(11). The potential of the excited state was supposed to be a screened and relaxed potential : we selected the $\mathrm{Z}+1$ atomic orbitals and removed one is electron to place it in the last occupied atomic orbital. We also performed calculations with the excited state chosen as being the same as the ground-state. The results of both calculations are quite similar, in 
agreement with the results obtained in the band approach of Müller et al, where the same potential is used for ground states and excited states $(5,6,12)$. We also tried a relaxed $\mathrm{Z}+1$ potential without screening where the sulfur atom was described with the chlorine orbitals in the $1 s^{2} 2 s^{2} 2 p^{6} 3 s^{2} 3 p^{4}$ configuration. The resulting spectra were not in good agreement with the experimental ones.

In formerly published calculations the energy positions of the resonances are well predicted by the MS theory but their intensities are much dependent on the muffin-tin radii(1). We chose them so as to minimize the potential discontinuity at the boundaries of the absorbing atom. Doing that we followed the prescriptions of Wille(13) instead of the more usual prescriptions of Norman(14). The potential discontinuities never exceeded two hundredths of a Rydberg between the sphere of the absorbing atom and its nearest neighbours. Nonetheless the main potential discontinuities between the muffin-tin spheres and the interstitial potential cannot be removed due to the muffin-tin approximation.

The MS theory has been developed with touching spheres though overlapping spheres should better describe the covalency by reducing the volume of the interstitial region. Actually calculations were performed with various overlap values from $0 \%$ to $30 \%$. We tried values of $0 \%$ and $10 \%$ in the calculations for small clusters. No significant differences were found so that we did not retain any overlap between adjacent spheres for the calculations in large clusters.

Therefore in the zincblende structure the choice of the muffin-tin radius of the absorbing atom determines nonambiguously the radii of all the other spheres in the cluster. The number of free parameters is limited in the way.

The exchange and correlation effects were taken into account through the density functional approximation. We used both $\mathrm{X}$-alpha and Hedin-Lundqvist potentials(15-16). For the X-alpha potential we made use of the alpha values tabulated by Schwartz(17). The $X$-alpha potential is energy independent and leads to good results for states near the vacuum level. The Hedin-Lundquist potential is an energy dependent complex potential, the imaginary part of which mimics the mean-free path of the scattered photoelectron.

\section{Results}

In semi-covalent tetrahedral semi-conductors the variations of the projected density of states of the photo-electron final state are responsible for the first features in the absorption cross section within $10 \mathrm{eV}$ from- the rising edge. In the highly symmetric environment of the absorber (the local symmetry group is $\mathrm{Td}$ ) these band effects are supposed to appear with an increasing size of the cluster. At both elements of $\mathrm{ZnS}$ we then studied the influence of the cluster size on the results. The convergence of the spherical harmonics developments were checked for all the spectra. All the following results have been obtained with $\mathrm{X}$ alpha potentials. This latter does not introduce any smoothing in its crude form and then the details of the calculations are sharper than the experimental spectra. To make a pertinent comparison with the experiment one should convolute the calculated spectrum with a lorentzian function, the width of which is to be the overall resolution. We have studied the influence of the cluster size. In pure $\mathrm{ZnS}$, for zinc and sulfur edges, the calculations have been performed for clusters size up to 99 atoms (eight atomic shells). The energy origin of all the figures has been taken at the interstitial muffin-tin potential for each calculation.

\section{Sulfur edge}

For light absorbers the high resolution allows to get tiny details in the absorption spectra but the features amplitudes decrease rapidly above the edge due to the low absorption cross-section for highenergy photoelectrons. We have calculated the full MS spectra of sulfur in $\mathrm{ZnS}$ in the first $40 \mathrm{eV}$ above the edge. Full MS events are indeed expected within that energy range.

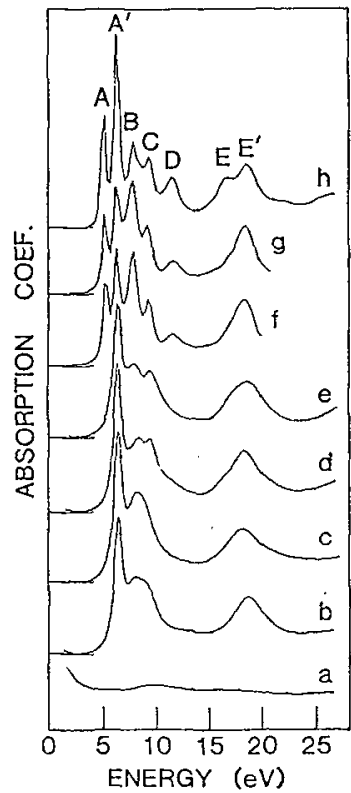

Fig. 1. X-alpha calculations for a sulfur atom in $\mathrm{ZnS}$ surrounded by its succesive shells of neighbours: a-one shell; b-two shells; c-three shells; d-four shells; e-five shells; f-six shells; g-seven shells; h-eight shells.

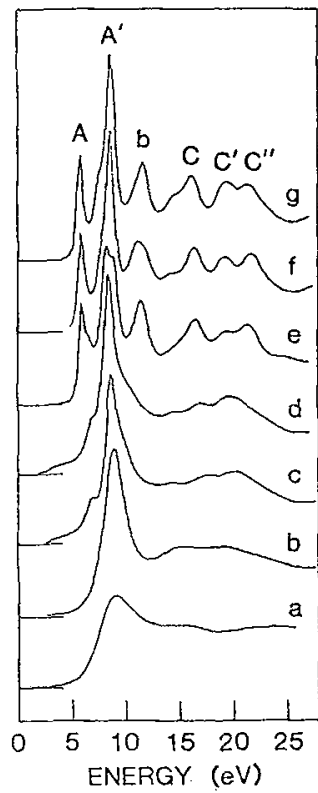

Fig. 2. X-alpha calculations for zine atom in $\mathrm{ZnS}$ surrounded $\mathrm{b}$ its succesive shells 0 neighbours: a-one shell; b-tw shells; c-three shells; d-fou shells; e-five shells; f-seve shells; g-eight shells. 
We increased the cluster size from the first shell of neigbours to the eighth shell. The eighth shell is at a distance of 14.46 u.a. from the central atom. In figure 1 we show the $X$-alpha calculations for the series of clusters. The main characteristic is the importance of the surrounding sulfurs in the scattering process. The addition of the zinc shells (shells of odd order) does not change much the calculated spectra while the addition of the sulfur shells always strongly modifies the spectra. This is to be expected on the basis that the zinc scattering amplitude is very small at low energy (less than 50 eV) similarly to what happens for copper $(18,19)$.

For sulphur K-edge the first shell of four zinc atoms does not reproduce the spectrum at all. The modulations obtained by the calculation cannot even be related to any resonance of the experimental spectrum due to the low scattering power of zinc. The first coordination shell is then absolutely unable to explain the experimental absorption spectrum.

With two and three shells (17 and 29 atoms) the main features are present except for the details before $10 \mathrm{eV}$ where MS events involving further shells are expected. Again from two to three shells no effects of the additional zinc shell is seen. With four shells ( 35 atoms) the resonance at $8 \mathrm{eV}$ is split giving the peaks labelled $B$ and $C$ in figure 1 . With six shells (71 atoms) a new resonance named D appears at $12 \mathrm{eV}$ and the first feature at $6 \mathrm{eV}$ is split in the two peaks $\mathrm{A}$ and $\mathrm{A}$ '. By taking into account the sixth shell the intensities of the features in the first $10 \mathrm{eV}$ change considerably. The last calculation has been performed with a cluster of eight atomic shells ( 99 atoms). The energy positions of the first resonances are identical to those for the six shell cluster but their intensities are different. The broad feature at $18 \mathrm{eV}$ is split into the two peaks $E$ and $E^{2}$.

Since the intensities of the features in the MS calculations are known to be dependent on the spheres overlap and on the muffin-tin radii, we have varied them up to $\pm 5 \%$ still keeping the spheres touching in the six-shell calculation. The relative intensities of the peaks $A, B, C$ and $D$ changed very little, leaving the spectra unchanged for energies higher than $10 \mathrm{eV}$. The shape of the spectra is instead much more dependent on the spheres overlap than on the muffin-tin radii. Calculations with the Norman prescriptions(14) which introduce spheres overlaps and consequent muffin-tin radii lead to changes in the relative intensities of about $10 \%$ without changing the overall shape. These calculations have been performed with screened and relaxed potentials for the excited state. To check this choice we performed calculations using the ground state potential in the description of the excited state as it is done for band calculations. Both results are almost similar.

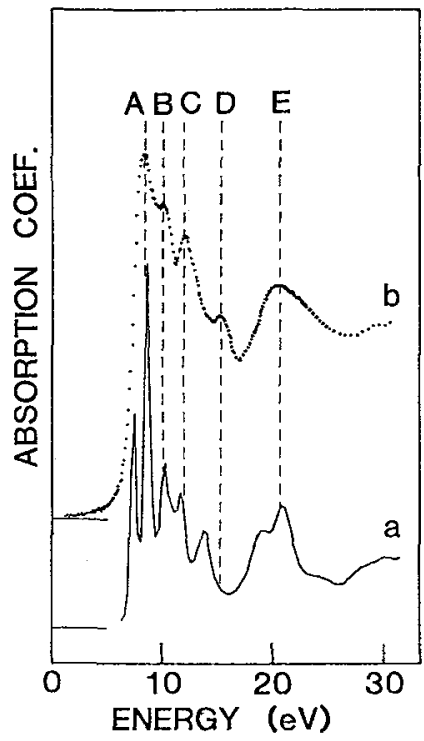

Fig. 3. a- experimental spectrum of sulfur atom $\mathrm{K}$-edge of $\mathrm{ZnS}$; $\mathrm{b}$ calculated X-alpha spectrum of a sulfur atom surrounded by 98 atoms (eight shells) in the $\mathrm{ZnS}$ structure.

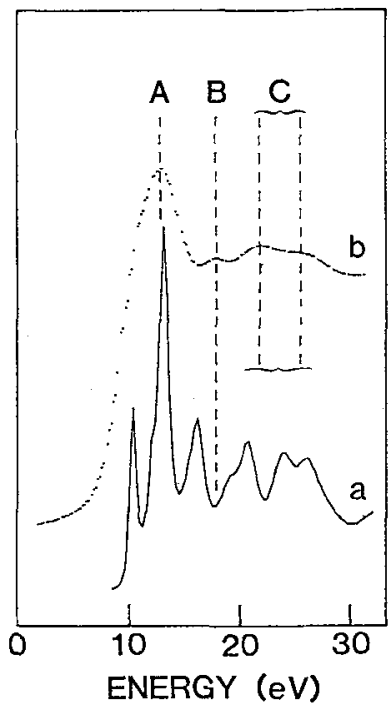

Fig. 4. a-experimental spectrum of the zinc atom K-edge of $\mathrm{ZnS}$; b-calculated X-alpha spectrum of a zinc atom surrounded by 98 atoms (eight shells) in the $\mathrm{ZnS}$ structure.

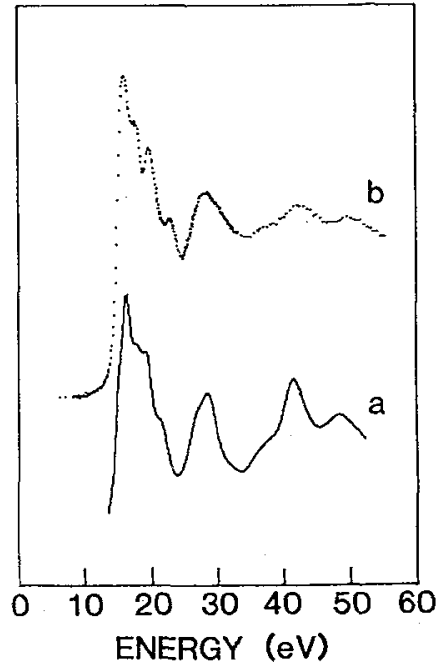

Fig. 5.Sulfur K-edge: a-X-alpha spectrum (99 atoms) convoluted by a Lorentzian of FWMH of leV; b-experimental spectrum. 
The comparison between the experimental spectrum and the eight-shell calculation is presented in figure 3 . The agreement is rather good. All the features present in the experimental spectrum $(A, B, C, D$ and $E)$ are reproduced by the calculation at the right energies but the splitting of both $A$ and $E$ peaks does not appear in the experimental spectrum. This is due to the broadening effects of the core level width and of the experimental resolution. We have convoluted the calculated spectrum by lorentzian and gaussian functions of different widths (0.8 to $1.2 \mathrm{eV}$ FWMH).If we convolute with a Lorentzian of FWMH of $1 \mathrm{eV}$ (figure 5), one obtain a good agreement for peaks A and $\mathrm{E}$ but on the other hand the peaks $\mathrm{B}$ and $\mathrm{C}$ are no more separated probably because of their slightly wrong intensities.

\section{Zinc edge}

We performed the same full MS calculations with clusters where zinc is the absorbing atom and studied again the influence of the cluster size. The calculations performed on sulfur guided our choices for the type of potential and the muffin-tin radii used. The calculations are presented in figure 2 .

For the first cluster with only a zinc atom at the center and four sulfurs the calculation already shows the gross features of the experimental spectrum (figure 4) due again to the big scattering power of sulfur atoms. As before in the following calculations the addition of sulfur atoms (shells of odd order) to the cluster give birth to new features while the addition of zinc atom shells (shells of even order) only slightly modifies them. The three-shell cluster (29 atoms) does not modify the main absorbtion peak $A$. The broad feature between $15 \mathrm{eV}$ and $20 \mathrm{eV}$ is separated in three peaks $\mathrm{C}, \mathrm{C}^{\prime}$ and $\mathrm{C}^{\prime \prime}$ present in the experimental spectrum but with different relative intensities.

By increasing the cluster size with the fifth shell ( 47 atoms) the peak $A$ is split in two parts. After convolution with a Lorentzian this result is consistent with the broad peak $A$ of the zinc experimental spectrum which exhibits a shoulder on the low energy side.

The introduction of the seventh shell ( 87 atoms) does not change drastically the calculated spectrum. It only enhances and sharpens the maxima. We also performed the calculation with an eight shell cluster ( 99 atoms). The eighth shell is made of 12 zinc atoms. As it has already been mentioned, they faintly contribute to the XANES spectra because of their low scattering amplitude. Actually this new calculation does not exhibit any major change.

It is to be noted that the calculation does not reproduce at the right energy the feature named $B$ in the experimental spectrum. The feature labelled $b$ in figure 2 which is present for clusters with five shells or more is at a too low energy to be assigned non-ambiguously to the $\mathrm{B}$ feature.

We can interpret the above results by saying that within $10 \mathrm{eV}$ above the edge band structure local density of state features are probed by the final state photoelectron due to the long mean free path in this insulating compound ( $\lambda$ about $25 \AA)$. However the experimental resolution plus the core hole lifetime correspond to a mean free path of the order of $12 \AA$ so that features up to the eighth shell $(2 R=14 \AA)$ can be resolved. On the contrary at higher energies plasmon-like excitations take place leading to a drastic reduction of the mean free path of the photoelectron $(5-6 \AA)$ so that only the second shell $(2 R=7 \AA)$ contribute to the absorption spectra (feature $E$ in figure 1 )

Reference

1-C.R. Natoli, D.K. Misemer, S. Doniach \& F.W. Kutzler: Phys. Rev. A 22, 1104 (1980); F.W. Kutzler, C.R. Natoli, D.K. Misemer, S. Doniach, \& K.O. Hodgson: J.Chem. Phys. 73, 3274 (1980)

2-S. Doniach, M. Berding, T. Smith \& K.O. Hodgson: in EXAFS and Near Edge Structure III, K.O. Hodgson, B. Hedman \& J.E. Penner-Hahn (eds) p 33 (Springer-Verlag, Berlin, 1984)

3-P.J. Durham, J.B. Pendry \& C.H. Hodges: Solid St. Com. 38, 159 (1981)

4-G.N. Greaves, P.J. Durham, G. Diakun \& P. Quinn: Nature 294, 139 (1981)

5-J.E. Müller \& J.W. Wilkins: Phys. Rev. B 29, 4331 (1984)-

6-J.E. Müller, O. Jepsen \& J.W. Wilkins: Solid St. Com. 42, 365 (1982)

7-D.D. Vvedinsky, D.K. Saldin \& J.B. Pendry: Surf. Scie. 156, 845 (1985); Surf. Scie. 162, 903 (1985); Surf. Scie. 162, 909 (1985)

8-F.W. Kutzler \& D.E. Ellis: Phys. Rev. B 29, 6890 (1984)

9-M. Kitamura, S. Murumatsu \& C. Sugiura: Phys. Rev. B 33, 5294 (1986)

10-P. Sainctavit, J. Petiau, A. Manceau, R. Rivallant, G. Renaud \& M. Belakhovsky: to be published

11-E. Clementi \& C. Roetti: Atomic Data Tables and Nuclear Data Tables 14 (1974)

12-L.A. Grunes: Phys. Rev. B 27, 2111 (1983)

13-L.T. Wille, P.J. Durham \& P.A. Sterne: J. Physique 47, C8-43 (1986)

14-J.G. Norman: Mol. Phys. 81, 1191 (1974)

15-S.H. Chou, F.W. Kutzler, D.E. Elllis, G.K. Shenoy, T.I. Morrison \& P.A. Montano: Phys. Rev. B.31, 1069 (1985)

16-L. Hedin \&B.I. Lundqvist: J. Phys. C Solid St. Phys. 4, 2064 (1971)

17-K. Schwartz: Phys. Rev. B 5, 2466 (1972)

18-J.E. Müller \& W.L. Schaich: Phys. Rev. B 27, 6489 (1983)

19-C.R. Natoli \& M. Benfatto: J. Physique 47 , C8-11 (1986) 